\title{
Pratiques
}

Linguistique, littérature, didactique

\section{Recherches en didactiques des mathématiques et du français : par-delà les différences}

\section{Claire Margolinas}

\section{(2) OpenEdition}

\section{Journals}

Édition électronique

URL : http://journals.openedition.org/pratiques/1491

DOI : 10.4000/pratiques. 1491

ISSN : 2425-2042

Éditeur

Centre de recherche sur les médiations (CREM)

Édition imprimée

Date de publication : 15 juin 2010

Pagination : 26-32

Référence électronique

Claire Margolinas, « Recherches en didactiques des mathématiques et du français : par-delà les différences », Pratiques [En ligne], 145-146 | 2010, mis en ligne le 15 juin 2010, consulté le 02 mai 2019. URL : http://journals.openedition.org/pratiques/1491; DOI : 10.4000/pratiques. 1491

(c) Tous droits réservés 


\section{Recherches en didactiques des mathématiques et du français : par-delà les différences}

Le lecteur de la revue Pratiques pourra être surpris de lire une contribution proposée par une didacticienne des mathématiques dans un volume consacré aux recherches en didactique du français... Dans un premier temps, j' expliquerai le contexte de cette intrusion $(\$ 1)$, puis je donnerai un aperçu de certains travaux issus de la didactique des mathématiques qui alimentent cette recherche $(\$ 2)$, je terminerai par quelques considérations sur les recherches en didactique(s) (\$3).

\section{Un objet de recherche, des approches}

\subsection{Des recherches sur les processus de production des inégalités scolaires}

Le travail auquel je me réfère dans cet article se situe dans le cadre du réseau RESEIDA $^{(1)}$ : lieu de rencontres et de recherches communes entre des didacticiens, des chercheurs en sciences de l'éducation, des sociologues et des psychologues, qui vise à structurer des recherches qui portent sur les processus de production des inégalités scolaires (Bautier, 2006). C'est dans ce cadre que se situent les recherches que je mène avec Marceline Laparra depuis 2004 (Laparra \& Margolinas, 2008, 2009, 2010 ; Margolinas \& Laparra, 2008, 2009a, 2009b).

Nous cherchons à élucider comment les processus de production des inégalités scolaires se constituent et se renforcent au sein même des interactions didactiques quotidiennes, à l'insu des acteurs.

Il n'est pas fréquent qu'un regard didactique soit porté sur une telle question, qui semble plus naturellement prise en charge par la sociologie de l'éducation, la sociolinguistique ou la psychologie sociale. Inscrire cette question dans le champ des didactiques, c'est considérer que la construction des connaissances et la transmission des savoirs, peuvent contribuer à la construction des inégalités scolaires ${ }^{(2)}$.

\subsection{Un ancrage dans la détermination des situations effectives}

Pour qu'un travail en commun, et non pas une simple juxtaposition, soit possible, il fallait que nos façons de concevoir l'analyse didactique soient compatibles, alors même que nos communautés disciplinaires ont des histoires et des paradigmes assez radicalement différents (Bronckart, 2005 ; Margolinas, 2005).

Cette compatibilité est obtenue grâce à un ancrage épistémologique fort dans nos disciplines respectives et une volonté et une capacité communes d'analyser les situations effectives des professeurs et des élèves.

En ce qui me concerne, cette capacité d'analyser les situations effectives était ma raison première pour participer au réseau RESEIDA. En effet, mes travaux depuis une quinzaine d'années (voir Margolinas, 1994, 2002, 2004) m'ont conduite à distinguer, dans une même classe et à un même moment, des situations différentes inves-

(1) Recherches sur la Socialisation, l'Enseignement, les Inégalités et les Différenciations dans les Apprentissages, dirigé par Jean-Yves Rochex et Elisabeth Bautier.

(2) Bien entendu, un phénomène d'une telle ampleur est sans aucun doute multiplement déterminé, notre ambition étant d'étudier la contribution apportée par des faits didactiques à ce phénomène et nullement d'affirmer que les didactiques peuvent seules expliquer celui-ci. 
ties par les élèves, à l'insu ou non du professeur. L'originalité de ce travail était de montrer qu'il s'agissait de différences didactiques c'est-à-dire que les connaissances investies par les élèves dans la situation et les connaissances potentiellement construites, pouvaient être très différentes selon les élèves et, surtout, très différentes de celles anticipées par le professeur et, le plus souvent, des savoirs institutionnalisés.

Ce travail est issu de la théorie des situations didactiques (Brousseau, 1998) et notamment d'un développement sur le concept de milieu et sa structuration (Brousseau, 1990). Le développement de la structuration du milieu comme une technique d'analyse des situations ordinaire m'a permis de différencier les situations suivantes : la situation envisagée par le professeur, les situations effectives qu'il installe et les situations investies par les élèves. Ce n'est pas si simple : en effet, le chercheur qui observe des classes ordinaires doit se déprendre d'une position de professeur qui le conduit soit à s'identifier à l'enseignant au point de ne pas voir les situations effectives installées à l'insu de l'enseignant, soit à s'envisager comme un conseiller qui se projette dans une autre situation potentielle envisagée a priori comme meilleure.

\subsection{La place des mathématiques et du français dans nos travaux}

Le travail pluridisciplinaire entre des didacticiens de formation différente est devenu assez courant depuis quelques années. Le plus souvent, il s'agit de recherches qui cherchent à déterminer ce qui est général, qui transcende les différentes disciplines, tout en gardant en ligne d'horizon les savoirs à transmettre (c'est le cas, par exemple, de la « didactique comparée », voir Mercier, Schubauer-Leoni, \& Sensevy, 2002).

Ce qui nous intéresse au contraire c'est de déterminer quels sont les savoirs en jeu, ce travail ne pouvant se réaliser que grâce à des connaissances disciplinaires et didactiques approfondies. En effet, nous ne nous contentons pas d'étudier ce qui est institutionnellement déclaré comme étant « du français » ou " des mathématiques ». Nous verrons dans la suite du texte et dans Laparra \& Margolinas (dans ce volume) que non seulement des connaissances linguistiques s'invitent dans des leçons déclarées être « de mathématiques », ce qui était prévisible, mais aussi que des connaissances mathématiques interviennent bien au-delà de leur périmètre institutionnel. Par ailleurs, nous cherchons à déterminer la nature des connaissances qui font obstacle à l'apprentissage, pour l'élève et à la compréhension des difficultés des élèves, pour le maître.

\section{Un savoir mis en évidence par des recherches en didactique des mathématiques}

\subsection{Relations entre recherches en didactique des mathématiques et savoirs mathématiques}

Les mathématiques représentent un champ de savoirs fort ancien et en constante évolution, dont une partie seulement est considérée par une société, à un moment donné de son histoire, comme étant à enseigner - à tous les citoyens, ou seulement à certains d'entre eux. En didactique des mathématiques, les travaux initiés par (Chevallard, 1985) sur la transposition didactique, développés ensuite par un très riche appareil théorique et méthodologique (Chevallard, 1992, 2003), ont pris comme objets d'étude les processus qui régissent les relations entre les différentes institutions (mathématiciens, concepteurs de programmes, etc.) qui conduisent à la détermination du savoir à enseigner.

Les recherches en didactique des mathématiques ne considèrent donc pas le savoir mathématique comme une donnée, dans le paragraphe qui suit, nous verrons qu'elle contribue également à identifier de nouveaux savoirs mathématiques. 


\subsection{L'énumération, un concept mathématique identifié par des recherches en didactique des mathématiques}

Le concept d'énumération a été décrit par Brousseau au début des années 80 , il a été l'objet du travail de thèse de Briand (voir Briand, 1999), il est actuellement l'objet de nouveaux développements (Margolinas, Rivière, \& Wozniak, 2010).

De quoi s'agit-il ? Pour dénombrer des points représentés sur une feuille, il faut connaître la comptine, mais aussi réaliser le geste suivant : désigner chaque point, une fois et une seule, jusqu'à ce qu'il n'y en ait plus. Briand (1999) a étudié cette connaissance et lui a donné un nom : l'énumération. Il faut organiser le parcours de la collection des points pour éviter de compter plusieurs fois un point ou d'en oublier un, ce qui est plus ou moins difficile selon la configuration (figure 1).
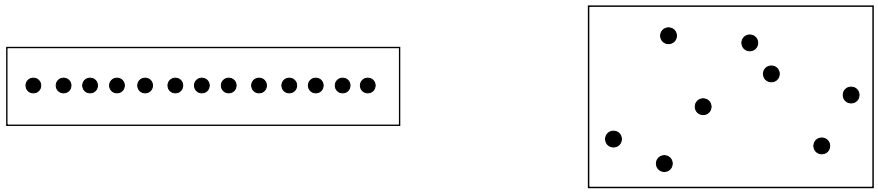

\section{Figure 1. Plusieurs configurations spatiales fixes}

De même, si l'on veut dénombrer des jetons, il faut déplacer les jetons déjà comptés au fur et à mesure de l'énoncé de la comptine. Il faut organiser spatialement la collection des jetons pour éviter de compter plusieurs fois un jeton ou d'en oublier (figure 2). Il s'agit aussi d'une énumération.

Cela paraît très simple dans le cas des jetons, le geste de pousser les jetons au fur et à mesure de l'énoncé de la comptine étant complètement naturalisé.

Si Briand s'est intéressé à l'énumération, c'est qu'elle pose de très nombreux problèmes aux élèves dès que les situations se compliquent : dénombrement d'objets dans des configurations « en désordre » ou mobiles (par exemple ballons), tri d'objets (voir infra).

Briand a montré qu'il existait des situations d'énumération sans dénombrement et que l'énumération

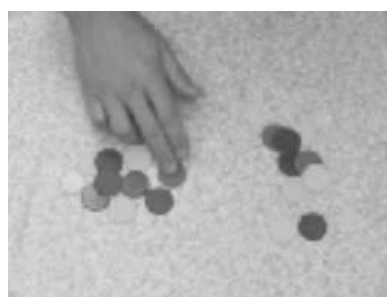

Figure 2. Une configuration spatiale variable était enseignable (Briand, Loubet, \& Salin, 2004). Il a montré également que l'on pouvait définir l'énumération mathématiquement, ce qui contribue à qualifier l'énumération comme un savoir.

\subsection{L'énumération : une difficulté persistante dans de très nombreuses activités}

Il y a de nombreuses activités durant lesquelles il faut énumérer des " collections ».

Dans le cadre de l'apprentissage de la lecture et de l'écriture, il est très courant d'avoir à manipuler des mots ou des lettres imprimés sur des étiquettes découpées, notamment en maternelle et au CP. Ces activités permettent en effet à l'enseignant de demander aux enfants de reproduire un modèle écrit, alors que leur graphie est encore malhabile.

Certains élèves réussissent très bien et rapidement ce type de tâche, alors que d'autres paraissent plus en difficulté, ce qui est interprété par le maître comme un problème dans l'identification des lettres. Or les difficultés d'énumération sont bien plus fréquentes et massives dans ce type d'activité que les difficultés de lecture.

Voici une observation en GS de maternelle qui illustre ceci. Dans le cadre d'un tra- 
vail autonome (la maîtresse est occupée par un autre atelier), les élèves avaient à leur disposition une bande alphabet d'étiquettes-lettres écrites en «script», un bol et une fiche (figure 3).

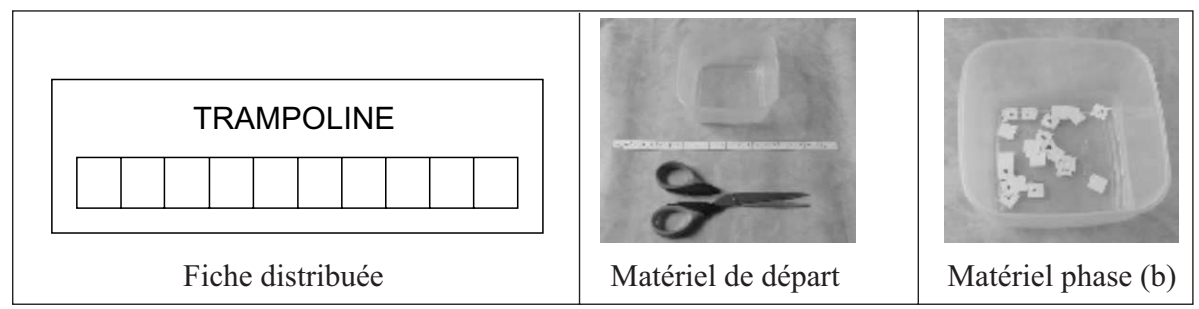

\section{Figure 3. Un exemple de travail en maternelle GS}

La maîtresse demande aux élèves (a) de découper les 26 étiquettes-lettres de l'alphabet et de les déposer dans le bol, (b) de choisir puis disposer les étiquettes-lettres dans les cases qui correspondent au mot (ici trampoline), (c) d'écrire « en attachée » le mot sur la ligne. Les élèves ont la possibilité d'utiliser un outil «alphabet » donnant la correspondance des graphies (bâton, script, écriture attachée), outil dont ils se servent de façon autonome très couramment dans cette classe.

Que se passe-t-il ? Les élèves découpent les étiquettes-lettres et les déposent dans la boîte, puis ils piochent dans la boîte, cherchent à reconnaître la correspondance entre la lettre piochée et la première lettre du mot modèle $(T)$ et, dans le cas où l'étiquette-lettre ne correspond pas à leur souhait, reposent celle-ci dans la boîte. Ce faisant, ils mélangent une étiquette déjà traitée et des étiquettes non encore traitées. À chaque «pioche », ils ont toujours une chance sur 26 de réussir... Aucun élève ne produit une organisation de la collection des étiquettes-lettres en plusieurs tas (traiténon traité, comme sur la figure 2). À la grande surprise de la maîtresse, ils n'ont souvent pas fini la tâche avec les étiquettes-lettres en écriture scripte alors qu'ils ont terminé celle d'écriture « en attachée » considérée comme de plus haut niveau. En effet, dans ce deuxième cas, pour écrire la première lettre, ils doivent repérer celle-ci sur le mot (T) puis l'écrire sur la ligne, s'ils ne connaissent pas cette graphie, ils la cherchent sur la règle-alphabet, simple énumération d'une collection fixe configurée en ligne, puis la reproduise, et ainsi de suite.

Pour appuyer cette observation, voici ce que fait Angélique, une des élèves les plus faibles de la classe, toujours un peu distraite. Elle n'a pas bien écouté la consigne. Au lieu de faire ce qui était demandé, elle a écrit le mot TRAMPOLINE dans les cases destinées à recevoir les étiquettes-lettres et n'a pas découpé la bande des lettres (figure 4). Elle s'aperçoit alors qu'elle n'a pas fait comme ses camarades et commence ensuite l'activité de reconnaissance et de collage des étiquettes-lettres dans les cases. Il lui suffit de reconnaître chaque lettre dans cette bande, de découper et de coller aussitôt chacune des lettres du mot modèle, ce qu'elle a réalisé aisément. Elle a fini bien avant les autres, démontrant ainsi que les difficultés rencontrées par ses camarades étaient des difficultés d'énumération.

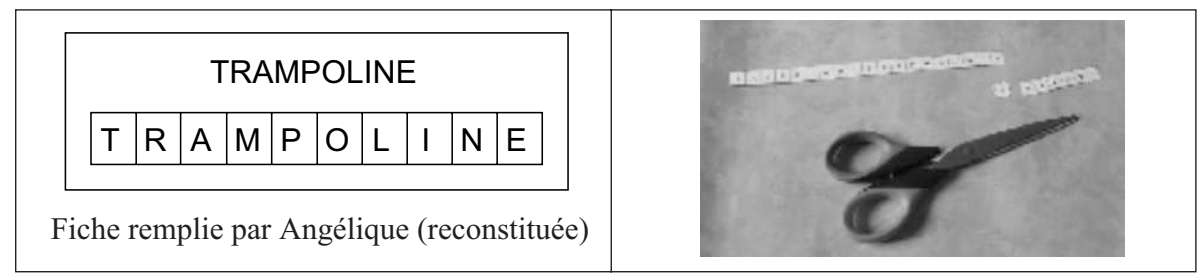

Figure 4. La situation d'Angélique 


\section{Recherches en didactique(s)}

\subsection{L'expérience des années 70}

Les travaux qui ont pris comme nom «didactique des mathématiques » prennent leur racines dans les années 60 , ils sont contemporains du mouvement des « mathématiques modernes " et de nombreuses personnes qui sont devenues ensuite des chercheurs en didactique des mathématiques ont participé au débat. La réforme des mathématiques modernes a été portée par des mathématiciens éminents, qui se sont intéressés à la diffusion des mathématiques, dans une optique conforme aux mathématiques développées au milieu du $20^{\mathrm{e}}$ siècle. La réforme des mathématiques modernes a été un échec social ${ }^{(3)}$ retentissant, et une contre-réforme a rapidement modifié les programmes.

La didactique des mathématiques s'est donc construite en France sur la certitude qu'un changement brutal, même bien intentionné, qui ne s'appuierait pas sur une connaissance approfondie des phénomènes de transmission des savoirs, serait inévitablement voué à l'échec.

\subsection{Recherches et didactique ? Recherches en didactique !}

Du fait des conditions historiques évoquées ci-dessus, la didactique des mathématiques s'est d'abord constituée au sein même des mathématiques ${ }^{(4)}$. Elle cherche à constituer un paradigme théorique original, indépendamment d'autres disciplines (comme la sociologie, la psychologie, l'histoire, l'anthropologie) et reconnu au sein des mathématiques. Elle a pour vocation, dès les années 70, d'être un champ scientifique au sens plein du terme.

Même si c'est plutôt la théorie mathématique qui fait notre force et notre notoriété, aussi bien auprès d'autres didactiques disciplinaires qu'au plan international ${ }^{(5)}$, la finalité sociale n'est pas absente et nos recherches investissent également le champ de l'application.

«La didactique des mathématiques se place ainsi dans le cadre des sciences cognitives comme la science des conditions spécifiques de la diffusion des connaissances mathématiques utiles au fonctionnement des institutions humaines.

Son but est essentiellement la connaissance d'une certaine catégorie de phénomènes, qu'il en découle ou non immédiatement des techniques, des décisions ou des moyens d'action. Ses rapports avec la technologie de l'enseignement sont celles d'une science avec ses applications.

Prise dans cette acception très générale, la didactique des mathématiques ambitionne de décrire les échanges et les transformations de savoirs à différentes échelles, aussi bien l'échelle des relations inter-culturelles du monde que celle d'une classe ou d'une leçon particulière.

Il s'agit là d'un projet très noble et très excitant. » (Brousseau, 1994 p. 52)

(3) Social, car nous ne pouvons pas dire, faute de données, s'il s'agit aussi d'un échec scolaire, c'est-à-dire si les élèves ont appris moins bien les mathématiques, quantitativement et qualitativement. Les textes de l'époque montrent d'ailleurs la colère des promoteurs de la réforme devant l'absence de formation des professeurs pour enseigner les mathématiques modernes.

(4) À l'heure actuelle, la majorité des enseignants-chercheurs en didactique des mathématiques appartient à la $26^{\mathrm{e}}$ section du CNU, celle des mathématiques appliquées, seule une minorité relève de la $70^{\mathrm{e}}$ section, c'est-à-dire des sciences de l'éducation.

(5) Guy Brousseau a été, en 2003, le premier récipiendaire du prix Félix Klein, attribué à un chercheur en didactique des mathématiques pour l'ensemble de son œuvre, par la Commission Internationale de l'Enseignement des Mathématiques. Yves Chevallard vient de recevoir (2010) le prix Hans Freudenthal, attribué par la même commission internationale pour un apport majeur dans ce champ de recherche. 


\subsection{Les champs disciplinaires en question}

L'exemple de l'énumération et son champ d'application hors des leçons qualifiées institutionnellement de mathématiques nous montre l'importance de recherches qui interrogent les qualifications disciplinaires de l'institution scolaire. Les recherches en didactique(s), parce qu'elles portent au centre de leur préoccupation la diffusion des savoirs et des connaissances, doivent pouvoir caractériser de l'intérieur ce qui relève ou non de leur champ.

Ainsi, les recherches en didactique(s) ont-elles peut-être intérêt à être moins frileuses (disciplinairement) : moins pour explorer le caractère général ou générique que pour questionner ce qui constitue le c?ur même des organisations de savoir.

Claire Margolinas, Université Blaise Pascal, Clermont-Ferrand, EA 4281, PAEDI, RESEIDA

\section{Références}

BAutier, E. (Ed.). (2006) : Apprendre à l'école, Apprendre l'école. Des risques de construction d'inégalités dès la maternelle. Lyon: Chronique Sociale.

BRIAND, J. (1999) : "Contribution à la réorganisation des savoirs prénumériques et numériques. Étude et réalisation d'une situation d'enseignement de l'énumération dans le domaine prénumérique ». Recherches en Didactique des Mathématiques, 19(1), 41-76.

BRONCKART, J.-P. (2005) : «Vingt-cinq ans de didactique de l'expression écrite. Eléments de bilan et perspectives d'avenir ». Revue suisse des sciences de l'éducation, 27(3), 361-380.

Brousseau, G. (1990) : «Le contrat didactique: le milieu ». Recherches en Didactique des Mathématiques, 9(3), 309-336.

— (1994) : «Perspectives pour la didactique des mathématiques ». In M. Artigue, R. Gras, C. Laborde, P. Tavignot \& N. Balacheff (Eds.), Vingt ans de didactique des mathématiques en France. Hommage à Guy Brousseau et Gérard Vergnaud. (pp. 51-66). Grenoble: La pensée sauvage.

- (1998) : Théorie des situations didactiques. Grenoble La Pensée Sauvage.

Chevallard, Y. (1985): La transposition didactique. Grenoble La Pensée Sauvage.

— (1992) : «Concepts fondamentaux de la didactique: perspectives apportées par une approche anthropologique ». Recherches en Didactique des Mathématiques, 12(1), 73-111.

- (2003) : «Approche anthropologique du rapport au savoir et didactique des mathématiques ». In S. Maury \& M. Caillot (Eds.), Rapport au savoir et didactiques (pp. 81-105). Paris: Fabert.

LAPARRA, M., \& MARGOLINAS, C. (2008) : Les premiers apprentissages de l'écrit : doxa et malentendus des écrits authentiques. Actes de Les didactiques et leur rapport à l'enseignement et à la formation, Bordeauxhttp://www.aquitaine.iufm.fr/infos/colloque2008/cdromcolloque/communications/lapa.pdf 
— (2009) : «Le schéma: un écrit de savoir? », Pratiques, 143-144(Numéro spécial: les écrits de savoir), 51-82.

— (2010) : «Situation et milieu, connaissance et savoir : des concepts pour la didactique des mathématiques et du français ». Pratiques.

Margolinas, C. (1994) : "Jeux de l'élève et du professeur dans une situation complexe ». Séminaire de didactique et technologies cognitives en mathématiques, Grenoble, from http://halshs.archives-ouvertes.fr/halshs-00418716

— (2002) : «Situations, milieux, connaissances : analyse de l'activité du professeur ». In J.-L. Dorier, M. Artaud, M. Artigue, R. Berthelot \& R. Floris (Eds.), Actes de la 11ème Ecole d'Eté de Didactique des Mathématiques (pp. 141-156). Grenoble La Pensée Sauvage.

— (2004) : «Points de vue de l'élève et du professeur : Essai de développement de la théorie des situations didactiques ». Habilitation à diriger les recherches en sciences de l'éducation. Université de Provence. , from http://tel.archives-ouvertes.fr/tel-00429580/fr/

- (2005) : «Essai de généalogie en didactique des mathématiques ». Revue suisse des sciences de l'éducation, 27(3), 343-360.

Margolinas, C., \& DE Redon, M.-C. (2008) : «Connaissances naturalisées dans le champ du numérique à l'articulation école maternelle / école primaire ». In A. Rouchier \& I. Bloch (Eds.), Perspectives en didactique des mathématiques (pp. cédérom). Grenoble: La pensée sauvage.

MARGOLINAS, C., \& LAPARRA, M. (2008) : Quand la dévolution prend le pas sur l'institutionnalisation. Actes de Les didactiques et leur rapport à l'enseignement et à la formation, Bordeauxhttp://www.aquitaine.iufm.fr/infos/colloque2008/cdromcolloque/communications/marg.pdf

— (2009a) : «Analyse de situations et production des inégalités scolaires ». Où va la didactique comparée? Genève, janvier 2009, from http://halshs.archivesouvertes.fr/halshs-00429565/fr/

— (2009b) : «Savoirs invisibles et connaissances cruciales : le cas des mathématiques en maternelle ». In C. Passerieux (Ed.), La maternelle. Première école, premiers apprentissages (pp. 99-107). Lyon: Chronique sociale.

Margolinas, C., RiviÈre, O., \& WozNiAK, F. (2010) : L'énumération : reprise en vue d'une diffusion aux professeurs des écoles et prolongements. Actes de Séminaire national de didactique des mathématiques, Paris

Mercier, A., Schubauer-LeOni, M.-L., \& SenseVy, G. (2002) : « Vers une didactique comparée ». Revue Française de Pédagogie, 141, 5-16. 